Hydrol. Earth Syst. Sci., 10, 469-484, 2006

www.hydrol-earth-syst-sci.net/10/469/2006/

(c) Author(s) 2006. This work is licensed

under a Creative Commons License.

\title{
Mapping mean and variance of runoff in a river basin
}

\author{
L. Gottschalk ${ }^{1}$, I. Krasovskaia ${ }^{1}$, E. Leblois ${ }^{2}$, and E. Sauquet ${ }^{2}$ \\ ${ }^{1}$ Department of Geosciences, University of Oslo, P.O. Box 1022 Blindern, 0315 Oslo, Norway \\ ${ }^{2}$ Cemagref, 3 bis quai Chauveau, CP 220, 69336 Lyon Cedex 09, France
}

Received: 8 December 2005 - Published in Hydrol. Earth Syst. Sci. Discuss.: 9 March 2006

Revised: 16 May 2006 - Accepted: 26 May 2006 - Published: 3 July 2006

\begin{abstract}
The study presents an approach to represent the two first order moments of temporal runoff variability as a function of catchment area and aggregation time interval, and to map them in space. The problem is divided into two steps. First, the first order moment (the long term value) is analysed and mapped applying an interpolation procedure for river runoff. In a second step a simple random model for the river runoff process is proposed for the instantaneous point runoff normalised with respect to the long term mean. From this model analytical expressions for the time-space variancecovariance of the inflow to the river network are developed, which then is used to predict how the second order moment varies along rivers from headwaters to the mouth. The observation data are handled by a hydrological information system, which allows to display the results either in the form of area dependence of moments along the river branches to the basin outlet or as a map of the variation of the moments across the basin. The findings are demonstrated by the example of the Moselle drainage basin (French part).
\end{abstract}

\section{Introduction}

Mapping statistical parameters of runoff across space constitutes one of the fundamental tasks in hydrology. As the probabilistic characteristics of the runoff formation process are a priori unknown, the only direct source of information for solving this task is hydrological observations series. Hydrological observation series, however, may be too few and/or too short for a reliable determination of quantitative runoff characteristics, or even not available at all. In this case, hydrology turns to indirect approaches for the study of runoff distribution in space, namely the "geographical interpolation" (in some sense) of the parameters of the runoff distribu-

Correspondence to: L. Gottschalk

(lars.gottschalk@geo.uio.no) tion. This is done utilizing hydrological series for different sites in a large river basin or different river basins within a region. Such studies fall under what is vaguely defined as "regional hydrology", to which this paper is a contribution.

The main hydrometerological processes (rainfall, evapotranspiration, temperature etc.) when observed at the land surface develop in a three-dimensional space - the two geographical coordinates $(x, y)$ and time $t$. The variation of these variables across space is described as contour (isoline) maps in classical works, i.e. they are space-filling phenomena and allow straightforward interpolation. River discharge (surface runoff), on the other hand, is formed in a two- dimensional space - the distance along a river $l_{A}$ (related to the point with area $A$ in a basin) and time $t$. This relationship to the area (and not the $(x, y)$ coordinates) explains why the variation of runoff characteristics across space, determined from discharge measurements, cannot be plotted as a contour map as simply as hydrometerological variables. Neither is it a space-filling phenomenon.

The contradiction in mapping runoff characteristics was noted early by Gergov (1972) with respect to the runoff "module". This latter concept, very often found in Central and East European hydrological literature, can have three interpretations -1 ) the true specific runoff $q_{1}$ with which a point $(x, y)$ in a basin contributes to the runoff in the river (rainfall excess; in the general case this might not be a space filling variable); 2) the areal mean runoff at a point $l_{A}$ in a river derived as $q_{2}=Q / A$ (where $Q$ is the mean annual discharge and $A$ the area of the corresponding basin); 3) the derivative of the specific runoff $q_{3}=d Q / d A$, i.e. the contribution of runoff to the river reach for each increment in the area. For all cases the dimension of runoff is volume per area and time $\left[\mathrm{L}^{3} /\left(\mathrm{L}^{2} \mathrm{~T}\right)\right]$, and in hydrology either " $1 / \mathrm{km}^{2} / \mathrm{s}$ " or " $\mathrm{mm} / \mathrm{year}$ " is used. Herein the latter of the two will be used.

This study presents an approach to represent runoff as a function of area (and thus on a map) in terms of how its statistical properties (mean value and variance) develop with

Published by Copernicus GmbH on behalf of the European Geosciences Union. 
area. The approach does not fully solve the theoretical ambiguities in mapping runoff but permits a reasonable simplification of the problem. We thus accept that runoff can be looked upon as a random variable in the two dimensional space $q=Q / A=q\left(l_{A}, t\right)=q(A, t)$. Consequently we accept the second definition given earlier, which also implies that runoff is only defined along a river network. Discharge measurements with modern technology represent almost instantaneous values for an instance $t$ in time. In practice, whether this technology is available or not, runoff characteristics represent a certain aggregation time interval $D$ (an hour, a day, five days, a month, a year). Mapping runoff characteristics involves thus mapping of statistical properties (moments) as a function of catchment area $A$ and duration $D-q=q(A, D)$ which is the focus for the present study. This task is similar to studying scale dependence in moments of runoff with respect to the area $A$ and duration $D$.

The long term mean value of runoff as well as the temporal variance show large variability across space revealing a nonhomogeneous random process. In general there exists a great coherence between the variability of these two moments i.e. a significant part of the variability of the variance might be explained by the pattern of variability of the mean. The mean value is independent of the time interval $D$ used, while this is not the case for the variance. The temporal variance decreases with time interval $D$. Furthermore, the variance decreases also with catchment area due to averaging over this area (the support of the random process). The full temporalspatial covariance structure of the runoff process needs to be known to be able to evaluate this variance reduction due to time and space averaging.

The patterns of spatial variability of the temporal mean and variance of runoff that can be identified from observations are of course influenced by the fact that these observations represent averaged values. To be able to map these quantities we need to assume the existence of an instantaneous point runoff (IPR) process. The task is thus to solve the inverse problem of identifying this process from the observations representing averages in time and space. In a second step we are able to average this IPR process along rivers to obtain the desired map.

For the long term mean runoff this task is rather straightforward and basically is a problem of stochastic interpolation with local support or, in other words, block kriging. An obstacle might be the complexity in the structure of the covariance of runoff as the data represents a mixture of nested and non-nested basins. This problem has been studied by Gottschalk (1993a) and the theoretical findings of this study will be brought forward here. Gottschalk (1993b) introduced a method for stochastic interpolation of runoff along the river network with a constraint preserving the water balance, i.e. at each downstream point in the river the runoff is the sum of the upstream inflow. Sauquet (2000) and Sauquet et al. (2000) developed this methodology further and combined it with a system for structuring hydrographical net- works in a hierarchical way called HydroDem (Leblois and Sauquet, 2000). It allows an effective reconstruction of the variation of mean annual runoff (first order moment) along the river network in a basin from discharge observations and a DEM. This latter interpolation scheme will be followed here to map the mean value. The resolution of the underlying DEM defines the size of the computational units (grid cells, sub-basins). It is further assumed that each unit contains a segment of a river ("a flow path"). The difference between the definition of runoff "module" (specific runoff) $q_{1}$ and $q_{2}$ is eliminated by this assumption and all the territory is assigned a runoff value down to the scale of basic computational units.

The patterns of spatial variability of the temporal variance identified from observations are still more complex than in case of the mean. There are two sources of variability. The first one is a reflection of the natural variability of the IPR process. This is then overlain by variability induced by the variance reduction which varies with the support i.e. the basin area. This part of the variability might constitute a significant part of the total variability. As such it introduces a dependence on the basin area. In principle, it would be possible to solve this problem also with stochastic interpolation with local support. A constraint can be added so that the sum of variance-covariances over sub-basins should add up to the total variance over the whole basin. The authors of this paper have made several attempts in this direction but they have all failed. The reason is mainly due to inconsistencies in observed data which do not satisfy the proposed constraint.

The alternative developed herein is as follows. Firstly we benefit from the fact that the pattern of the mean value is also reflected in the variance by introducing a new variable namely the normalized instantaneous point runoff i.e. IPR divided by the long term mean at a point. For this latter variable a parametric random model is proposed that allows constructing a space-time covariance structure for the runoff along rivers and specifically mapping the variance along rivers.

The paper is structured as follows. In the first section of the paper, the statistical characteristics of runoff data from the Moselle basin (France) are described which are used to test the approach. In the next section the method for interpolation of the mean is described and a random model for river runoff in a basin is proposed which allows to estimate variance functions, reduction factors, and auto- and crosscorrelations. The derivation of these latter functions is rather laborious and is only briefly presented in an Appendix. The derived theoretical expressions are then used to model the spatial and temporal scale dependence of the first and second order moments of temporal variability, the autocorrelation at a site, and the cross-correlation between sites along rivers of the Moselle basin. Examples of derived maps of mean annual runoff and the coefficient of variation of runoff of different durations are shown in an Appendix. The paper ends with a discussion of the results and conclusions. 


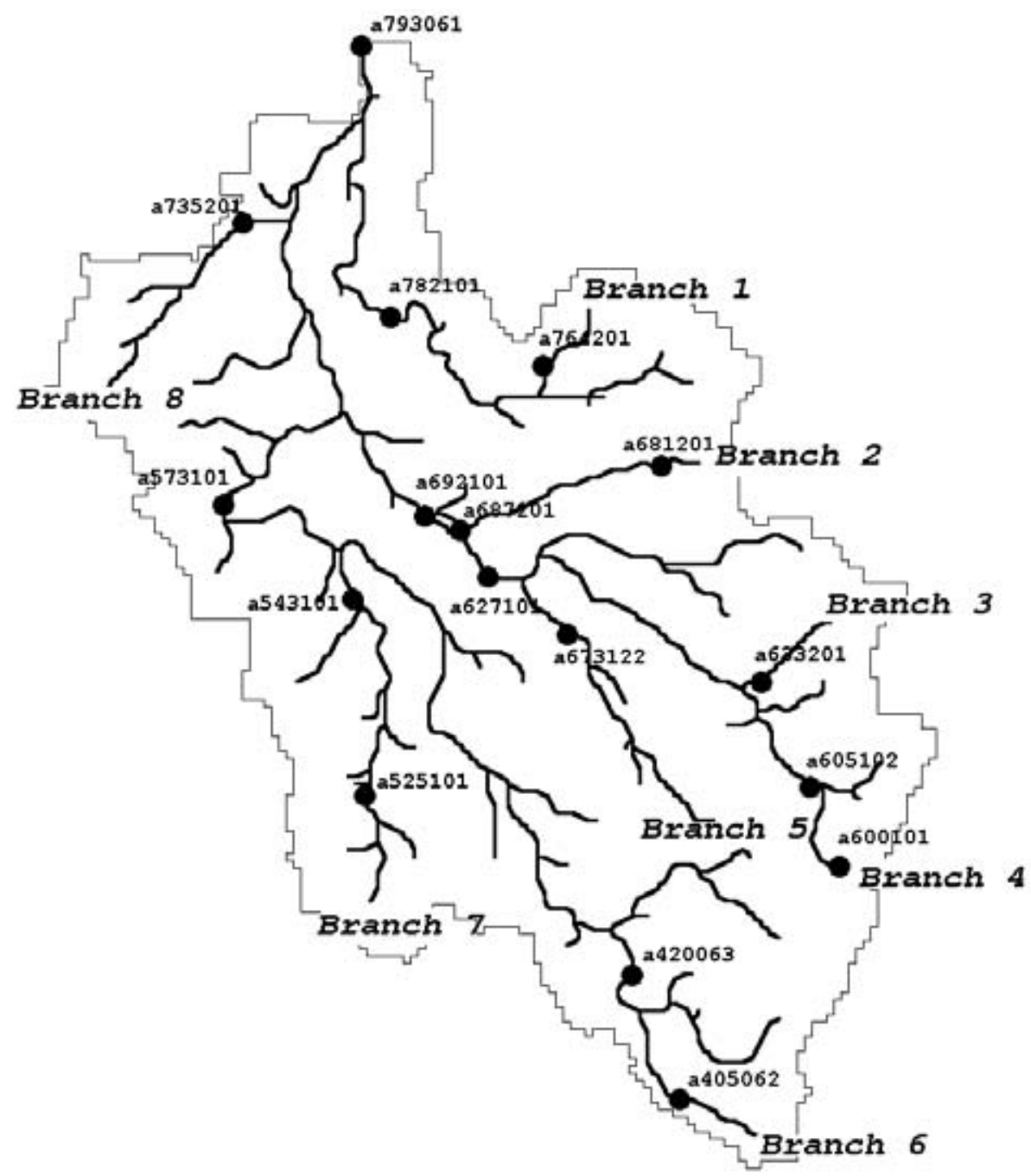

Fig. 1. Schematised river network of the Moselle basin down to Hauconcourt (a793061) with eight main branches and 17 gauging stations used in the study.

\section{Runoff characteristics of the Moselle River basin}

The study area covers the French part of the Moselle basin, one of the main tributaries to the Rhine River. The Moselle River basin demonstrates a great variability of the landscape due to the heterogeneity of the geology (crystalline and sedimentary rocks, schist, sandstone) and relief under continental climate. The headwaters are located in the Vosges Mountains covered by forests whereas the downstream parts are lowlands (alluvial plains) with a landscape influenced by agricultural practices.

A map of the Moselle basin and the drainage pattern identified by HydroDem is shown in Appendix A. The resolution of the map is $1 \mathrm{~km}^{2}$, which is the size of the grid cells used for producing maps of the runoff characteristics. This map of the Moselle basin is complemented by a schematised river network with its eight main branches as well as the discharge stations (Fig. 1). The size of the main basin is $9387 \mathrm{~km}^{2}$ at its outlet at Hauconcourt, France. Within this basin another 16 sub-basins with areas in the range of $73-3350 \mathrm{~km}^{2}$ have been utilised. Data for a common period 1970-1997 have been used. Not all stations had complete records for this period. Stations with a record of at least 15 years were included in the study. The discharge data used are breakpoints and thus allow the estimation of runoff averaged over any duration $D$. Here five durations have been considered, namely: an hour, a day, five days, a month and a year.

The study focuses on the two first order moments of runoff variability in time. By this we accept the concept of "partial characterization" of complex variation patterns and of a sequential analysis of variability (Gottschalk, 2005). Characterization by the one dimensional distribution function is the first step. In a characterisation by distribution function in the general case a multivariate distribution would be needed for a 


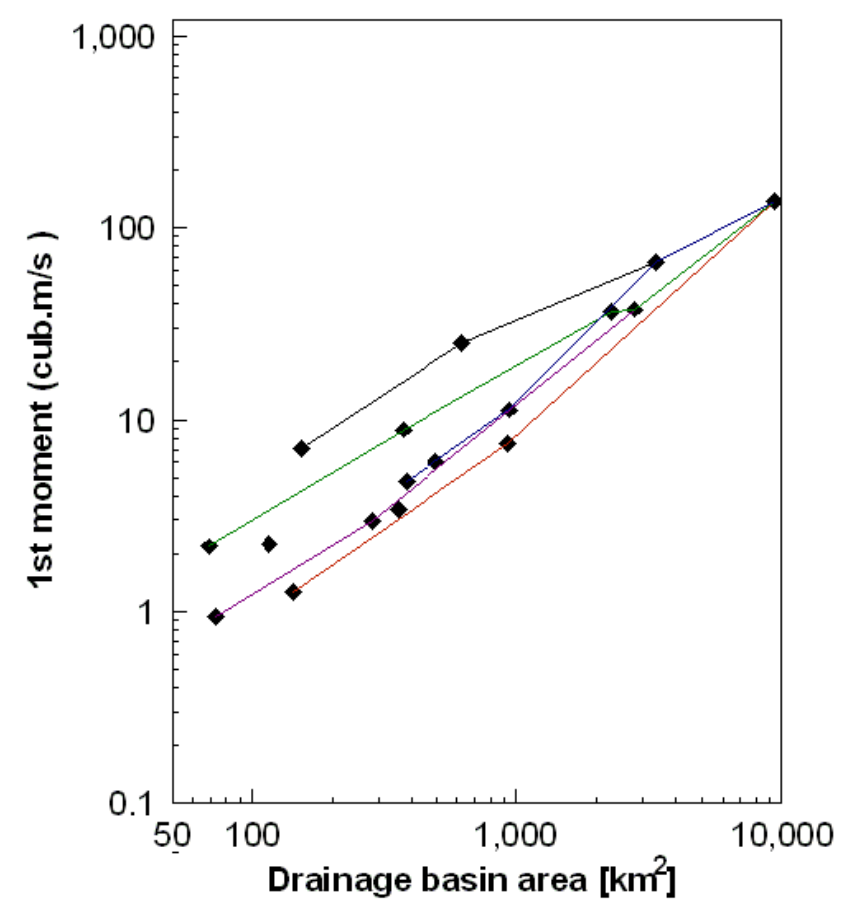

obs. br1 br2 br4 br6 br7

Fig. 2. Estimated first moment of runoff (i.e. long term mean runoff) of the Moselle stream gauges.

complete characterisation. The one dimensional distribution constitutes in this case the marginal distribution of the data, which is the same as the flow duration curve widely used in hydrology. We thus choose to study the two first moments of this marginal distribution constructed from data averaged over different durations and for the time being do not consider the structure of this variability over time, for instance the role of seasonality.

The first order moment of the Moselle discharge data are plotted against basin area in a double logarithmic diagram in Fig. 2 in accordance with the traditional way of representing scale dependences. There is a tendency that the data cluster around straight lines but the scatter is quite large. The first order moments (the long term mean values) do not depend on duration $D$ but higher order moments do. This spatialtemporal dependence for the second order moment is illustrated in Fig. 3 in terms of the coefficient of variation. For short durations (an hour and a day) this coefficient shows a strong decay with the area. This dependence on area decreases with the increase in duration and for annual values it is negligible. For small catchments the estimated values differ between one hour and one day duration. This difference almost disappears for basins bigger than $1000 \mathrm{~km}^{2}$.

Figure 4 shows the empirical auto- and cross-correlations at and between runoff stations along the tributary to the

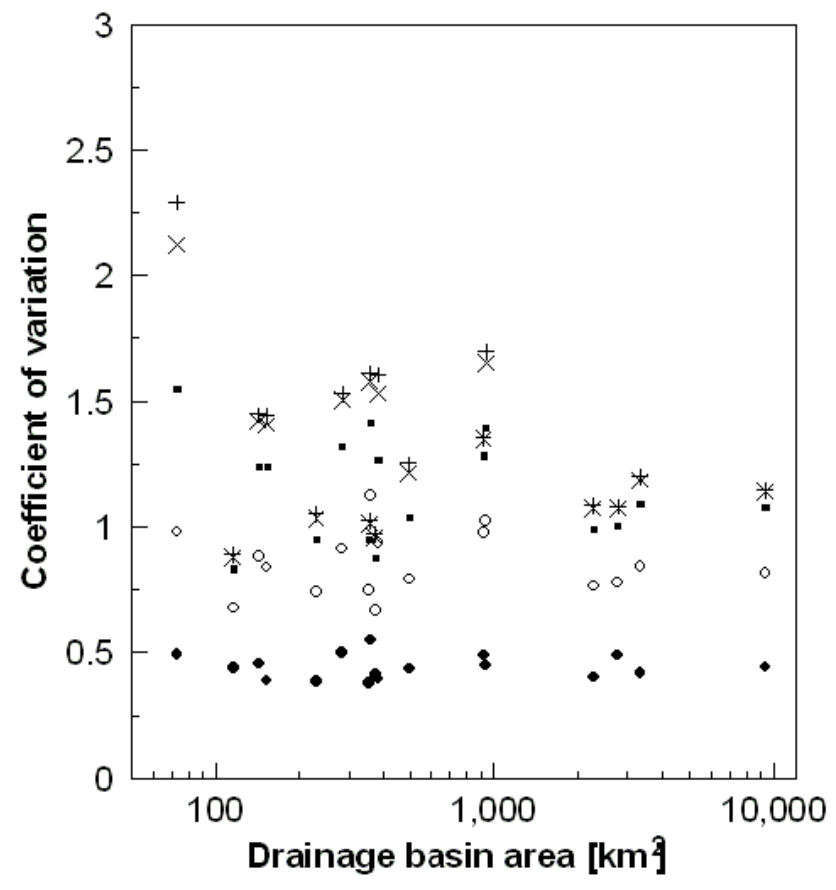

$\begin{array}{ccccc}\text { 1h } & 1 \mathrm{~d} & 5 \mathrm{~d} & 30 \mathrm{~d} & \text { year } \\ + & \times & \bullet & \circ & \bullet\end{array}$

Fig. 3. The estimated temporal coefficient of variation of runoff for the Moselle discharge data plotted against basin area $A$ for different durations $D$. This normalised second order moment decreases with the increasing duration from an hour (the highest value) to a day, five days, a month and a year (the lowest value).

Moselle viz. the Meurthe River (branch 4, cf. Fig. 1) and the outlet site of the Moselle River at Hauconcourt (station a793061). It shows the estimated autocorrelation function for the central site in this river branch (station a627101) for the four different durations considered (a), the same function for one hour duration for the five sites along the same river branch (b), the cross-correlation between the central and outlet site for different durations (c) and the cross-correlation between the site at the outlet and those upstream (d).

There is a strong autocorrelation in the data over small time lags (hours, days). The decay of the correlation for larger time lags is not of the exponential type frequently used in hydrology. A heavy tail is observed. We interpret this as the existence of (at least) two characteristic scales - one of the order of one day and another of the order of one month. Examining the autocorrelation at a site (Fig. 4a) we note that the correlation increases with increasing time interval from hours, to days, five days and months. One would expect a similar although less pronounced behaviour when moving downstream and the basin size is increased (Fig. 4b). For very small time steps (hours) this is actually the case. However, the tail of the autocorrelation function does not show any clear systematic pattern corresponding to the size of the 

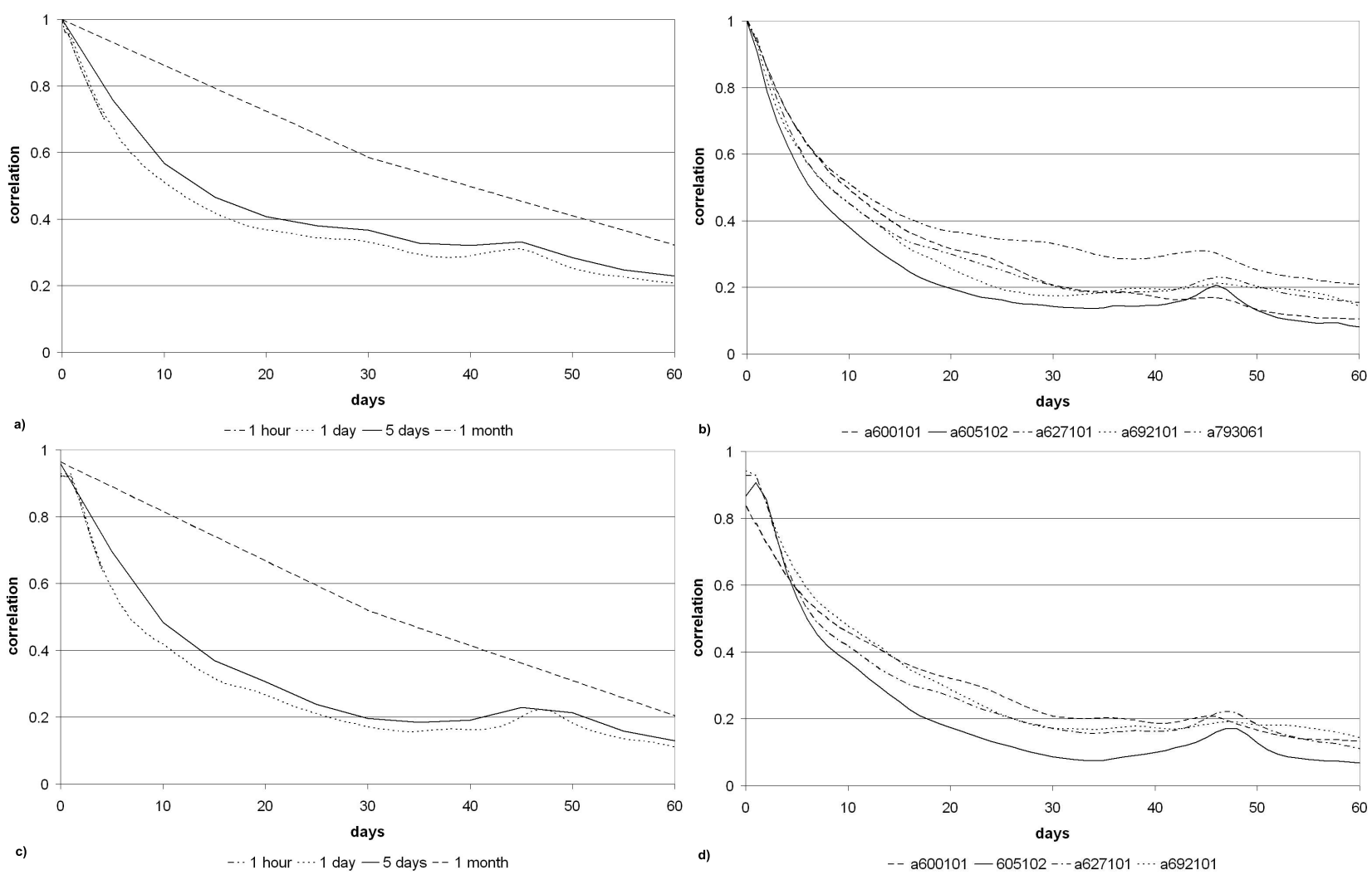

Fig. 4. Estimated empirical correlation functions for five sites on branch 4 (the Meurthe River) down to the outlet (station a793061): (a) the autocorrelation function for the central site (a627101) for different durations; (b) the same function for one hour duration for the five sites along branch 4; (c) the cross-correlation between the central and outlet sites for different durations and (d) the cross-correlation between the outlet and upstream sites for daily data. (In a and $\mathrm{c}$ the curves for $1 \mathrm{~h}$ and 1 day coincide except for very short time lags and the former is therefore hidden behind the second one).

basin, although there is a slight tendency of an increased memory when moving downstream along the branch. The impression is that the weight of the heavy tailed part of the autocorrelation varies locally.

The empirical cross-correlation functions show a drop ("nugget") in the correlation at lag zero, which is larger the larger the difference in basin size is. At a site this drop decreases with the increasing duration (Fig. 4c). However, the behaviour at short time lags can be quite complex - sometimes with a small increase before decaying and in other cases an immediate decay (Fig. 4d). For the time being it has not been possible to explain all the details in the behaviour of the correlation functions.

The spatial correlation, revealed in Fig. $4 \mathrm{~d}$ for individual sites, would allow to show the plot of spatial correlation coefficients along and between river branches against some distance measure. The structure of such a diagram is, however, quite complex. Runoff as related to points along rivers is a non-homogeneous process (Gottschalk, 1993a). Furthermore, nested and non-nested sub-basins show drastic differences in correlation, very high in the first case and lower in the other. Finally, the distance measure is not obvious in this case. Should it be Euclidian distance between observation sites, distance between the centres of gravity of the basins, distance along the rivers between the sites etc.? Whatever the measure used, the result is a scatter of points without any structure.

\section{Long-term mean annual runoff}

Let $X\left(l_{A}, t\right)$, the inflow to a river at a length coordinate $l_{A}$ and time $t$, represent a two dimensional random field. We allow the long term mean value $m_{X}\left(l_{A}\right)$ to vary in space $l_{A}$ but let it be constant in time $t$. We thus admit that the runoff production systematically from one locality to another in a basin, i.e. the generic process is non-homogeneous in space. On the other hand, stationarity is postulated in time. In reality runoff shows seasonal variations with more or less stable patterns (Krasovskaia and Gottschalk, 1992). We will anyhow accept this as a simplification for the time being as was already commented on in the previous section. 


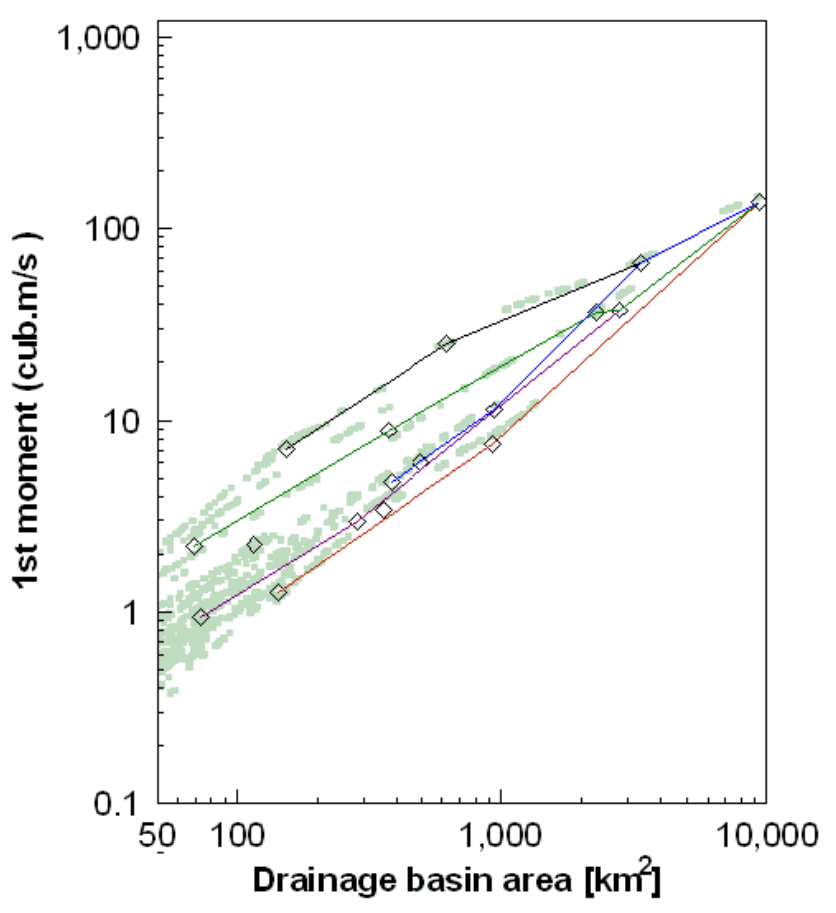

map obs. br1 br2 br4 br6 br7

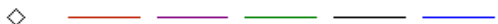

Fig. 5. Estimated first moment of runoff (i.e. long term mean runoff): Diamonds are the local estimates from the stream gauge data, grey points are the mapped values along the streams.

The long term mean value $m_{X}\left(l_{A}\right)$ characterises the variation in the intensity in runoff formation across space being a product of the local landscape and climatic features. This first order moment is not influenced by the dynamics of the runoff formation process as the higher order moments are. We are thus able to map $m_{X}\left(l_{A}\right)$ with a due consideration of the fact that it is a variable in a one dimensional space $l_{A}$, the coordinate along the river network, applying stochastic interpolation with local support. We will follow here the approach developed by Sauquet et al. (2000). First, a theoretical model of an assumed point process is estimated from the empirical covariance function. This point covariance function is then used for interpolation of runoff to each grid cell of the Moselle basin following a hierarchical scheme to be able to satisfy a water balance constraint along the river.

The covariance between two sites in a nested river system was earlier treated by Gottschalk (1993a). A simple exponential function is postulated for the correlation of the inflow to the river between to points $l_{A 1}$ and $l_{A 2}$ along the river at a distance $\lambda=\left|l_{A 1}-l_{A 2}\right|: \rho(\lambda)=\exp (-\lambda / K$. The constant $K$ has the dimension of length and describes the characteristic scale of variation of runoff formation and reflects landscape and long-term climatic features. An approximate expression for a corresponding covariance between two nested basins is derived as:

$$
\begin{aligned}
\operatorname{Cov}\left(A_{1}, A_{2}\right)= & \operatorname{Cov}\left(L_{A 1}, L_{A 2}\right) \\
= & 2 \sigma_{x}^{2}\left(\frac{K^{2}}{L_{A 1} L_{A 2}}\right)\left\{\frac{L_{A 1}}{K}+\frac{1}{2} e^{-L_{A 1} / K}\right. \\
& +\frac{1}{2}\left(1-\frac{L_{A 1}}{K}+\frac{L_{A 2}}{K}\right) \\
& \left.\left(e^{-L_{A 2} / K}-e^{-\left(L_{A 2}-L_{A 1}\right) / K}\right)-\frac{1}{2}\right\}
\end{aligned}
$$

$A_{1}$ and $A_{2}$ denote the two areas of sub-basins, where the first is nested within the second, drained by the river segments of lengths $L_{A 1}$ and $L_{A 2}\left(L_{A 1}<L_{A 2}\right)$, where the shorter one is common. Let us specifically look at the situation when $L_{A 1}=L_{A 2}=L_{A}$, i.e. we get an expression for the variance over the sub-basins. Insertion into the equation above yields:

$\operatorname{Var}(A)=\sigma_{A}^{2}=2 \sigma_{x}^{2}\left(\frac{K}{L_{A}}\right)^{2}\left\{\frac{L_{A}}{K}-1+e^{-L_{A} / K}\right\}$

Whether we use area or distance the expression for the covariance between two sub-basins along the same river anyhow indicates a non-homogeneous process, i.e. it depends on the absolute coordinates $A_{1}\left(L_{A 1}\right)$ and $A_{2}\left(L_{A 2}\right)$ and not on the relative difference between the positions of the points with these coordinates. The covariance along a river is thus represented by an ensemble of curves conditioned on the position of the downstream site $A_{2}\left(L_{A 2}\right)$ as a function of the difference in area (position). For the covariance between sites in non-nested basins it is not possible to develop close form analytical expressions, but it must be derived numerically as a conventional covariance with local support.

The details of parameter estimation and interpolation follow Sauquet et al. (2000). The characteristic space scale was estimated as $K=50 \mathrm{~km}$ (Eq. 1) and the spatial point standard deviation as $\sigma_{X}=300 \mathrm{~mm} /$ year. The resulting dependence with basin area is shown in Fig. 5 and the corresponding map in Appendix A. It would be possible to show a space filling map of runoff. We prefer to show runoff in the map only along main rivers $\left(A>50 \mathrm{~km}^{2}\right)$ so that the map better reflects the actual variability covered by available observations.

Figure 5 shows the long term mean runoff estimated from the discharge records as diamonds. The HydroDem software (Leblois and Sauquet, 2000) utilised in this study, allows a retrieval of a unique string that arranges the grid cells on a map in accordance with the structure of the river network, thus linking the position of the grid cell with the integrated area of the basin at this position. Accordingly, we are able to plot interpolated runoff at each grid cell of the map against area which is shown as a grey background in the figure. When scale dependence is studied in the context of a river basin the scale relations develop along river branches towards a common value at the outlet (this is guaranteed due to the water balance constraint in the interpolation scheme). The diagram in Fig. 5 reveals the relative contribution from the branches 
to the total discharge. The flow in the main river can therefore be higher (lower) than the surrounding contribution due to high (low) inflow upstream.

\section{Normalised instantaneous point runoff}

In a second step we now turn to the second order moments with the assumption that the variation in $m_{X}\left(l_{A}\right)$ is known. To achieve homogeneity in space the original process is normalised with respect to this long term mean:

$Z\left(l_{A}, t\right)=X\left(l_{A}, t\right) / m_{X}\left(l_{A}\right)$

This homogeneous random field in time and space $Z\left(l_{A}, t\right)$ is the main focus for the study with the aim of developing a random model for this variable and specifically deriving expressions for its variance-covariance function.

For the general formulation of a time-space process Vanmarcke (1988) distinguishes between three important special types of two dimensional covariance functions, namely:

- the covariance structure is separable

- the correlation structure is isotropic, i.e. the covariance structure can be expressed in terms of the "radial" covariance function

- the covariance structure is ellipsoidal, i.e. by appropriate scaling and rotation of the coordinate axes random fields with ellipsoidal covariance structure can be reduced to isotropic random fields.

Gandin and Kagan (1976) suggest a covariance model similar to the second type for use in meteorology and climatology:

$\operatorname{Cov}[\lambda, \tau]=\sigma^{2} \rho(|(\lambda / v)+\tau|)$

where $\lambda$ as before is the relative distance between two points in the river, $\tau$ is the time lag, $\rho()$ is a one dimensional correlation function to be specified, $v$ is a velocity and $\lambda / v$ can be interpreted as a time of travel. The expression has its root in the so called Taylor's hypothesis for turbulent flow also known as the Taylor "frozen turbulence" hypothesis. We will here assume that the process $Z\left(l_{A}, t\right)$ has this type of isotropic space-time covariance function.

$Z\left(l_{A}, t\right)$ describes the instantaneous inflow at a point in a river. The important variable is the integrated process $\bar{Z}(A, t)$, i.e. the normalised discharge of a river with a basin of size $A$. We derive it by integration of $Z\left(l_{A}, t\right)$ from the most distant point in the river $\left(l_{A}=0\right)$ down to its outlet at $l_{A}$. In accordance with the formulation of the covariance function Eq. (4) we replace the distance by the time of travel (time of concentration) $T_{C A}=L_{A} / v$ along the river distance $L_{A}$. The integrated process is thus expressed by:

$\bar{Z}(A, t)=\bar{Z}\left(T_{c A}, t\right)=\frac{1}{T_{c A}} \int_{t-T_{c A}}^{t} Z\left(t^{\prime}, t\right) d t^{\prime}$

$$
=\frac{1}{T_{c A}} \int_{t}^{t+T_{c A}} Z\left(t-t^{\prime}\right) d t^{\prime}
$$

This formula coincides in form with a system interpretation of the rational method (Singh, 1988, p. 123) if runoff is expressed in terms of flow per area, i.e. a unit pulse of duration $T_{c A}$ and depth $1 / T_{c A}$. There is of course an important difference in the fact that we here deal with the inflow to the river and not directly with rainfall like in the rational method. It is a simplification that neglects the dynamics and non-linearity of river flow. The only argument for doing this is the Principle of Parsimony as formulated by Tukey (1961): It may pay not to try to describe in the analysis the complexities that are really present in the situation. He stresses the importance of reconsidering a model structure towards a simpler representation, which might improve the performance of the estimation method.

The mean value of the process $\bar{Z}\left(T_{c A}, t\right)$ is independent of time of concentration because the aggregation is linear and it is unity as it represents a normalised value (see Eq. 3). The variance-covariance, however, will change with changing time of concentration. The covariance function for the integrated process is derived as:

$$
\begin{aligned}
& \operatorname{Cov}\left(\bar{Z}\left(T_{c A}, t\right), \bar{Z}\left(T_{c A}, t+\tau\right)\right) \\
& =\operatorname{Cov}_{A}(\tau)=\frac{1}{T_{c A}^{2}} \int_{t}^{t+T_{c A}} \int_{t+\tau}^{t+T_{c A}+\tau} \sigma_{Z}^{2} \rho\left(t^{\prime}-t^{\prime \prime}\right) d t^{\prime} d t^{\prime \prime}
\end{aligned}
$$

This type of double integral can be easily transformed to a single integral by a simple variable transformation (e.g. Gottschalk, 1993a). A more general method for simplification is to apply the following relation between the covariance for areas (lines as special cases) and the underlying point covariance (Matérn, 1960):

$$
\operatorname{Cov}_{A}(\tau)=\int_{\min (h)}^{\max (h)} \operatorname{Cov}(|h|) f(h) d h=E[\operatorname{Cov}(|h|)]
$$

where $f(h)$ is the probability density function of distances $\mathrm{h}$ between two points chosen at random within the two line segments that are separated by a specified distance $\tau$. For the special case of $\tau=0, f(h)$ is the density function of all possible distances within a line segment of length $T$ which has the well known expression: $f(h)=\frac{2}{T}\left(1-\frac{h}{T}\right)$. The distribution function for a more general case of random distances between two line segments of equal length $T$ shifted by the distance $\tau$ is derived using general results by Ghosh (1951):

$$
\begin{array}{ll}
f_{1}(h)=\frac{1}{T_{c A}}\left(1+\frac{h-\tau}{T_{c A}}\right) ; & \left(\tau-T_{c A}\right) \leq h \leq \tau \\
f_{2}(h)=\frac{1}{T_{c A}}\left(1-\frac{h-\tau}{T_{c A}}\right) ; & \tau<h \leq\left(\tau+T_{c A}\right)
\end{array}
$$


When integrating this distribution in accordance with Eq. (7) three cases have to be distinguished namely i) $\tau=0$; ii) $0<\tau \leq T_{c A}$; and iii) $\tau>T_{c A}$ :

i) $\tau=0$;

$$
\begin{aligned}
\operatorname{Cov}_{A}(0)=\operatorname{Var}_{A}= & \sigma_{Z}^{2}\left\{\int_{-T_{c A}}^{0} \rho(-h) f_{1}(h) d h\right. \\
& \left.+\int_{0}^{T_{c A}} \rho(h) f_{2}(h) d h\right\} \\
= & 2 \sigma_{Z}^{2} \int_{0}^{T c A} \rho(h) f_{2}(h) d h
\end{aligned}
$$

ii) $0<\tau \leq T_{c A}$;

$$
\begin{aligned}
\operatorname{Cov}_{A}(\tau)= & \sigma_{Z}^{2}\left\{\int_{-\left(T_{c A}-\tau\right)}^{0} \rho(-h) f_{1}(h) d h\right. \\
& +\int_{0}^{\tau} \rho(h) f_{1}(h) d h \\
& \left.+\int_{\tau}^{T_{c A}+\tau} \rho(h) f_{2}(h) d h\right\}
\end{aligned}
$$

iii) $\tau>T_{c A}$;

$$
\begin{aligned}
\operatorname{Cov}_{A}(\tau)= & \sigma_{Z}^{2}\left\{\int_{\tau-T_{C A}}^{\tau} \rho(h) f_{1}(h) d h\right. \\
& \left.+\int_{\tau}^{\tau+T_{c A}} \rho(h) f_{2}(h) d h\right\}
\end{aligned}
$$

Let us exemplify the derivations above by assuming a simple exponential correlation function:

$\rho(h)=\exp (-h / k)$

where $\mathrm{k}$ is a time constant. The following expression is derived:

i) $\tau=0$;

$$
\begin{aligned}
\operatorname{Cov}_{A}(0)= & \gamma(A)=\sigma_{A}^{2}=2 \sigma_{Z}^{2}\left(\frac{k}{T_{c A}}\right)^{2} \\
& \left\{\frac{T_{c A}}{k}+e^{-T_{c A} / k}-1\right\}
\end{aligned}
$$

i) $0<\tau \leq T_{C A}$;

$$
\begin{aligned}
\operatorname{Cov}_{A}(\tau) & =2 \sigma_{Z}^{2}\left(\frac{k}{T_{c A}}\right)^{2}\left\{\frac{T_{c A}-\tau}{k}\right. \\
& \left.+\frac{1}{2}\left(e^{\left(\tau-T_{C A}\right) / k}+e^{-\left(\tau+T_{C A}\right) / k}\right)-e^{-\tau / k}\right\}
\end{aligned}
$$

iii) $\tau>T_{c A}$;

$$
\operatorname{Cov}_{A}(\tau)=\sigma_{Z}^{2}\left(\frac{k}{T_{c A}}\left\{1-e^{-T_{c A} / k}\right\}\right)^{2} e^{-\left(\tau-T_{C A}\right) / k}
$$

The time constant $k$ characterises the scale of persistence in the inflow to the river system. This constant thus describes the dynamics of the runoff process in contrast to the constant $K$ introduced in Eq. (1), which describes the characteristic spatial scale of variation in runoff formation. The two limiting cases of Eqs. (11a-c) are when $k \rightarrow 0$ and $k \rightarrow \infty$, respectively. In the first case the covariance function Eq. (9) turns into a Dirac's delta function $\delta(\lambda)$ for $\lambda=0$, i.e. the characteristic of a process without memory. Applying the relationships Eqs. $(11 \mathrm{a}-\mathrm{c})$ to this situation we find:

i) $\tau=0$;

$$
\operatorname{Cov}_{A}(0)=\sigma_{A}^{2}=\sigma_{Z}^{2} / T_{c A}
$$

ii) $0<\tau \leq T_{c A}$;

$$
\operatorname{Cov}_{A}(\tau)=\sigma_{Z}^{2} \frac{T_{c A}-\tau}{T_{c A}^{2}}
$$

iii) $\tau>T_{C A}$;

$$
\operatorname{Cov}_{A}(\tau)=0
$$

i.e. a moving average process over a time period $T_{c A}$. For the second case with a very large memory ( $k$ large compared to $T_{c A}$ ) the variance is constant equal to $\sigma_{Z}^{2}$.

Equation (11a), with Eq. (12a) as a special case, is thus the variance function for instantaneous runoff from an area of size $A$. It describes how the variance of runoff changes with this size (time of concentration $T_{c A}$ ). Taking the square root of this expression yields the corresponding reduction factor. In a system terminology it represents a combination between linear reservoirs entering into a linear channel or as first order autoregressive processes combined with a moving average one. Equations $(11 \mathrm{~b}, \mathrm{c})$ and $(12 \mathrm{~b}, \mathrm{c})$ represent the auto-covariance functions of instantaneous runoff of a basin of size $A$.

Hydrological data of runoff often represents averaged values for a day or some other duration and the derived expressions are not directly applicable as they concern instantaneous runoff. To make them compatible we need to integrate these equations in the time domain over a duration 
of $D=1$ day. We will omit detailed derivations in the text as the principle is the same as described earlier, i.e. using Eq. (7) develop frequency functions of distances between line segments (river branches, time intervals) $f(h)$ and then integration schemes in accordance with Eq. (8). The resulting equations of such derivations are shown in Appendix B for the variance functions for a basin of size $A$ and duration $D$ (Eq. B1), the auto-covariance function for a basin of size $A$ and time lags $n D, n=0,1, \ldots$ (Eq. B2), and the crosscovariance function between two areas with sizes $A 1$ and $A 2$ and time lags $n D, n=0,1, \ldots$ (Eq. B5). Some intermediate results are also shown. To simplify the notation, dimensionless variables $\delta=D / k$ and $\eta=T_{c A} / k$ replace duration $D$ (time scale) and $T_{c A}$ (space scale).

The random model, in principle, allows a reproduction of all statistical characteristics of the runoff data from the Moselle basin referred to in the first section of this paper. The theoretical model shows how they are interrelated. The parameters of the model can be determined by fitting the respective theoretical function to the empirical ones, established from the observed data. We will concentrate these efforts to the variance function (Eq. B1), which expresses the dependence of the variance on the time of concentration $T_{c A}$ (area) and duration $D$. The other functions will be used as a diagnostic test of model assumptions.

\section{Fitting the model for the normalized variable}

The second order moment of the normalized variable $Z$ equals the coefficient of variation squared of the original variable $X$. Figure 3 showed the dependence of the coefficient of variation on area $A$ estimated from the observed runoff data for different durations $D$ (an hour, a day, five days, a month and a year). In case of so-called simple scaling this parameter should be constant (Gupta and Waymire, 1990) but the pattern of variation seen is indeed complex. In principle we should be able to model the behaviour in accordance with the variance function (Eq. B1), depending on the dimensionless variables $\delta=D / k$ and $\eta=T_{c A} / k$, respectively, and containing one parameter $\sigma_{Z}$ equal to the coefficient of variation for instantaneous inflow to a river branch. Following the development along different branches a mirrored pattern of the variation of the first order moment (although with an increased scatter) can be identified, i.e. high runoff values show low coefficients of variation and vice versa. It was also found that the decay in the time dependence was poorly described by the assumed simple exponential function for the instantaneous point correlation function (Eq. 11). Two time scales could be identified (cf. Fig. 4) - one on the order of a day and another on the order of a month. To account for this fact, the point exponential correlation function is modified as $\rho(h)=w \exp \left(-h / k_{1}\right)+(1-w) \exp \left(-h / k_{2}\right)$, where $w$ is a weight coefficient. The variance function (Eq. B1) needs to be modified accordingly. As all operations for the derivation of this function from the point correlation function are linear the modified variance function is derived as two weighted components as in Eq. (B1) with weights $w$ and $(1-w)$ and with time variables $\delta_{1}=D / k_{1}, \delta_{2}=D / k_{2}$ and time of concentration variables $\eta_{1}=T_{c A} / k_{1}$ and $\eta_{2}=T_{c A} / k_{2}$, respectively.

For the variables $\eta_{i}, i=1,2$ and the point standard deviation $\sigma_{Z}$ the following relations are proposed to take account of the dependence on basin area and mean runoff, respectively:

$$
\begin{aligned}
\eta_{1} k_{1} & =\eta_{2} k_{2}=T_{c A}=a+b \ln (A) \\
\sigma_{Z} & =c\left(1+d \times m_{X}\right)
\end{aligned}
$$

It is assumed that the time of concentration increases linearly with the logarithm of the basin area $A$ and that the coefficient of variation increases or decreases linearly with the mean annual runoff $m_{X}$.

We thus obtain a model for the description of the dependence of the coefficient of variation on basin area $A$ and duration $D$ containing seven parameters, namely $w, k_{1}, k_{2}$, $a, b, c, d$. Some of these parameters might vary with location, and should therefore develop along river branches. Here we for the time being assume a set of global parameters for the whole Moselle basin and determine them so that an optimal fit in the least square sense is obtained with the scatter of data in Fig. 6. A downhill simplex method (Press et al., 1992, p. 326-330) was applied for the search of optimal parameters, with the following result: $w=0.8$ [-], $k_{1}=0.63$ [days], $k_{2}=40.0$ [days], $a=-10.4$ [days], $b=2.77$ [days $\left./ \ln \left(\mathrm{km}^{2}\right)\right], c=2.7[-], d=0.012\left[(\mathrm{~mm} / \text { year })^{-1}\right]$. The derived relations are not applicable to resolve variability for small basins $\left(A<43.5 \mathrm{~km}^{2}\right)$. The absolute estimation error in the coefficient of variation obtained for this set of parameters was 0.18 , to be compared with the standard deviation of this coefficient 0.47 . The explained variance is thus 0.82 . The model is best in explaining variance between the different durations, while the explained variance across different sites in space amounts to 0.5 . Most stations follow the general pattern except for one outlying observation station in the Vosges Mountains (a405062).

The estimated parameters confirm the impression of a decrease in the coefficient of variation with the increase of the mean runoff and the existence of two characteristic time scales of the correlation function one a little less than a day and the other about one and a half months. The parameters allow us to estimate the coefficient of variation as a function of basin area $A$ and duration $D$. The resulting relationships are shown in Fig. 6 where observed values for basins are compared with those estimated. The coefficient of variation has also been estimated for each grid cell in the digital map. Each grid cell defines an upstream catchment area and a mean runoff to be used in the empirical formulas. Only values for an area larger than $50 \mathrm{~km}^{2}$ are considered as in case of the map of the long-term mean runoff. These estimated values are shown as a grey background on the graphs. The proposed model is able to reproduce the main features 
1 houl

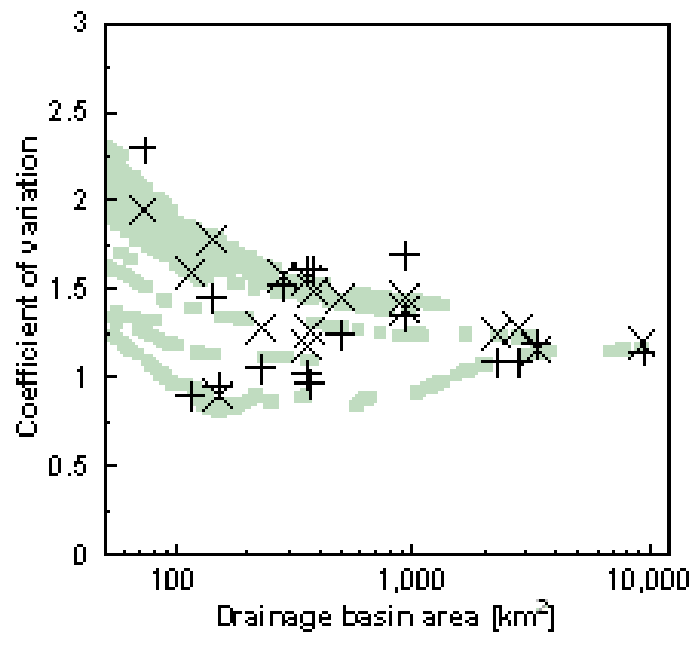

5 days
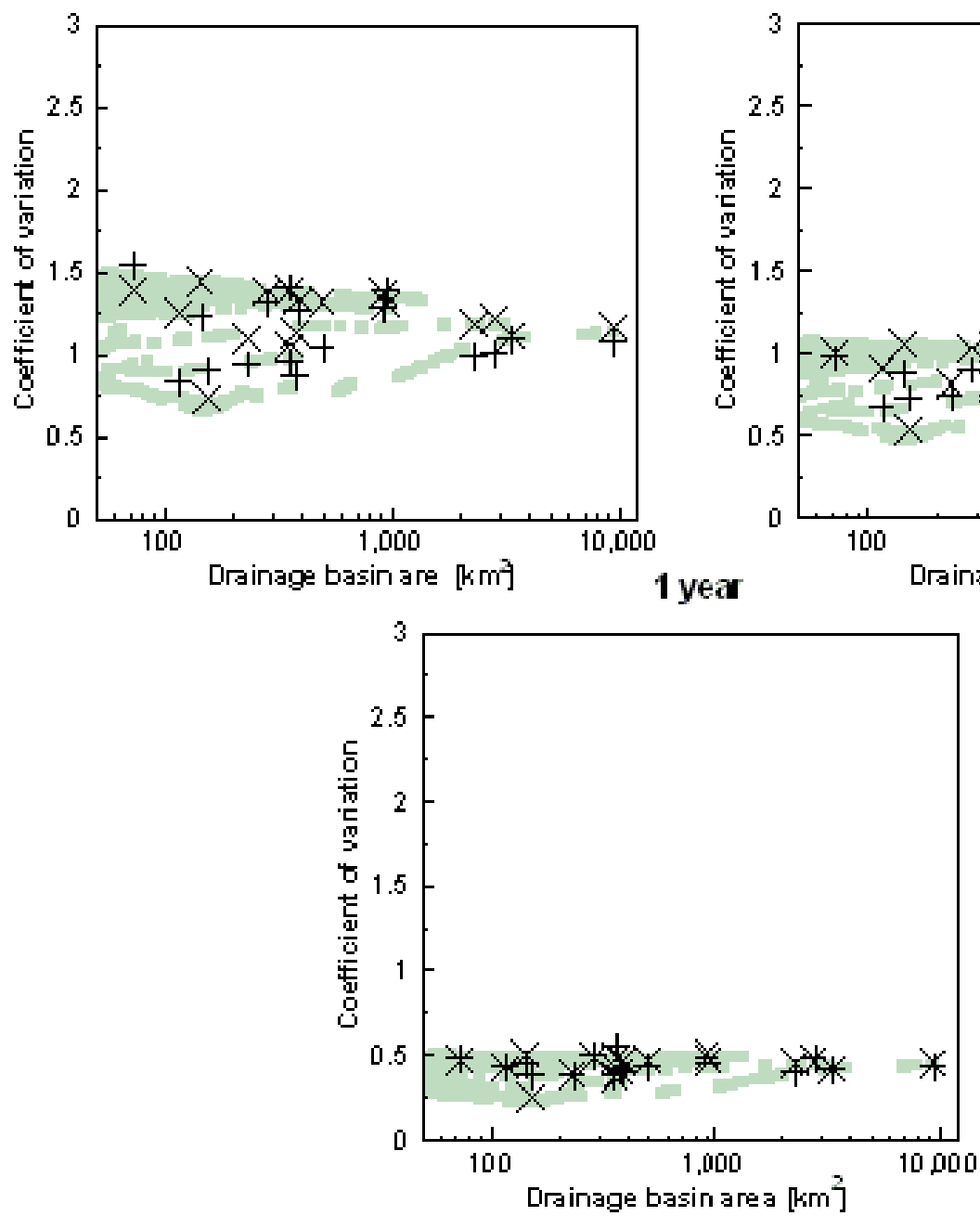

1 day

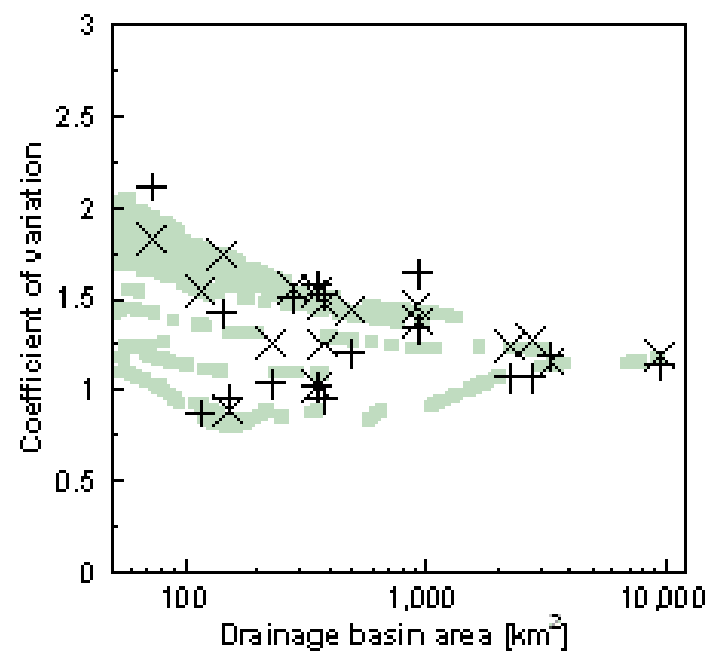

30 days

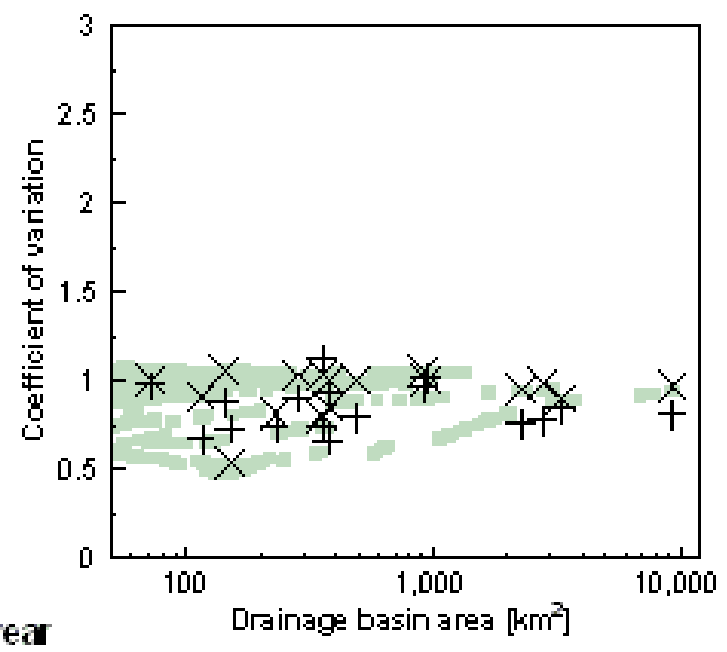

Fig. 6. Temporal coefficients of variation of runoff. Plusses are the estimates from local stream gauge data; crosses are the estimates of the stochastic model for the gauged sites; grey points are the estimates of the stochastic model for the grid cells of the DEM (see Appendix A). 

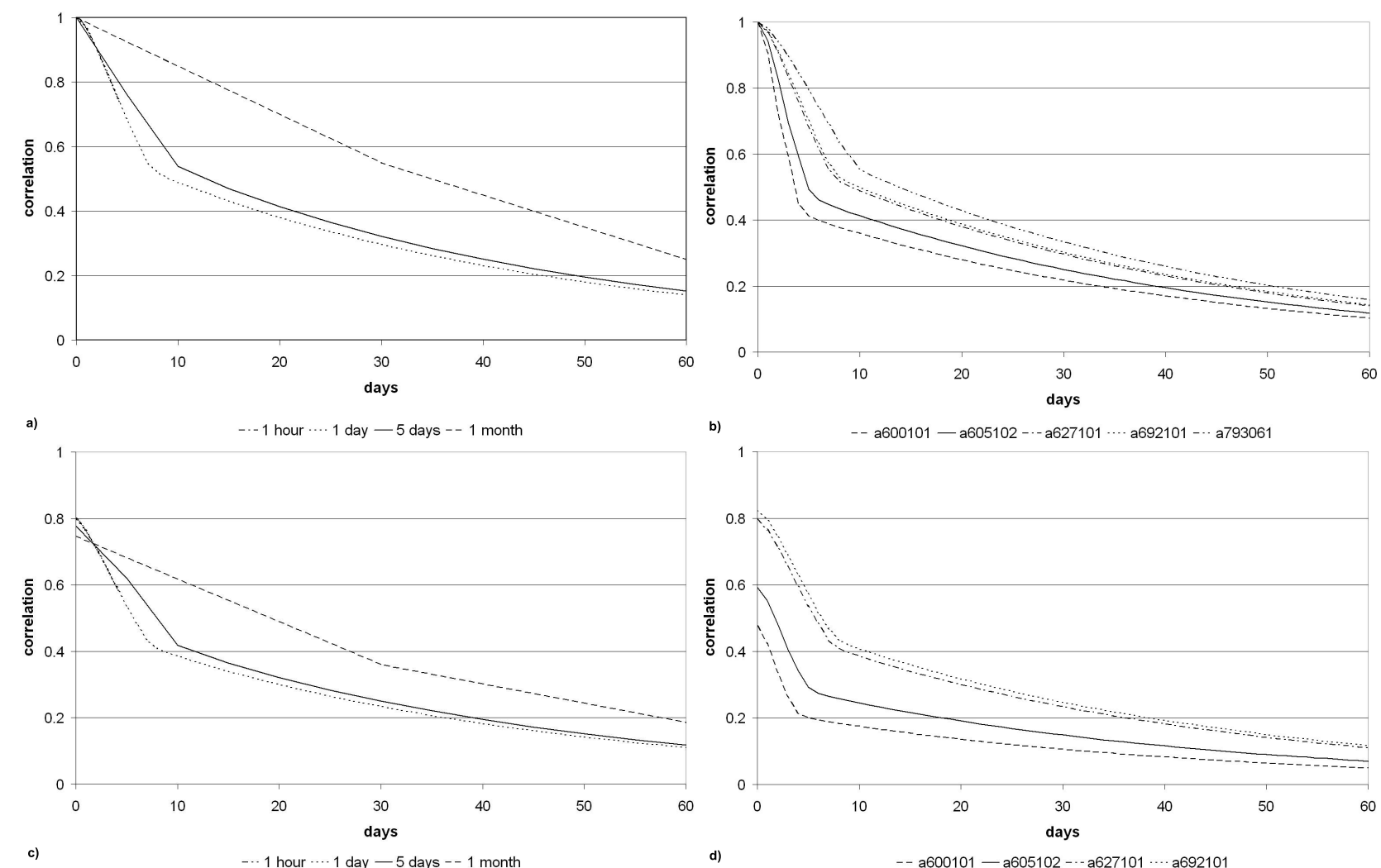

Fig. 7. Modelled correlation functions for five sites on the same river (branch 4): (a) the autocorrelation function for the central site in this river branch for different durations; (b) the same function for one hour duration for the five sites along the same river branch; (c) the cross-correlation between the central and outlet sites for different durations and (d) the cross-correlation between the outlet and upstream sites for daily data. The corresponding empirical functions are illustrated in Fig. 4. for daily data. (In a and c the curves for $1 \mathrm{~h}$ and 1 day coincide except for very short time lags and the first one is therefore hidden behind the second one).

of the dependence of the coefficient of variation $C_{v}$ on both the area and the duration. The corresponding maps can be constructed and an illustration for $C_{v}$ for $D=1$ day and $D=1$ month is given in Appendix A.

\section{Testing auto- and cross-correlation}

The approach developed here is based on the existence of a covariance function along the river network. We have here chosen to determine the basic parameters in this covariance structure through the dependence of the coefficient of variation on the area $A$ and duration $D$. It thus remains to be shown that the main features of this covariance structure are reproduced. Here we will do this by comparing the empirical statistical properties shown earlier in Figs. 4 a to $4 d$ and those obtained by the model with the parameters determined in the previous section. The covariance includes duration as well as area, which would mean that we should be able to model the autocorrelation at a site in a river for different durations
$D$, as well as the cross-correlation between sites along a river branch.

A comparison of empirical and model correlation functions carried out here should not be seen in the light of a formal test. The theoretical derivations developed herein are in its infancy and not yet ready for such formal procedures. This comparison is rather to be seen as a first diagnostic to indicate if the assumptions make sense. We will use branch 4 as an illustration when comparing the theoretical derivation with the empirical functions of Fig. 4. Figure 7a thus shows the estimated autocorrelation (Eq. B2) for durations of an hour, a day, five days and a month for the central site on this branch, and Fig. $7 \mathrm{~b}$ the autocorrelation function for one day duration for all stations along the branch. Figure $7 \mathrm{c}$ in a similar way shows the estimated cross-correlation function (Eq. B5) between the central and the outlet sites for different durations and Fig. 7d the cross-correlation function between the outlet site and upstream stations along the same river branch for a duration of one day.

In general the agreement is acceptable. The empirical and modelled correlation functions show the same main features. 
There are of course details both at very small and large time scales that are not yet captured by the model. It describes well the behaviour of the empirical autocorrelation functions for different durations in time. However, the model is not able to reproduce the details of the spatial unsystematic dependence of the empirical correlation functions along branches. The deviation between the model and empirical functions is largest in small basins. This can, to a certain extent, be traced back to the empirical relationship of the time of concentration variable to $\ln (A)$, which contains a lower bound $\left(\sim 43 \mathrm{~km}^{2}\right)$.

We note that it was necessary to compose the theoretical model of two parts - one for small and the other for large time lags represented by the two parameters $k_{1}$ and $k_{2}$, respectively and a weight coefficient $w$. For the time being, we assumed these parameters to be the same for the whole of the Moselle basin. For larger sub-basins this can be an acceptable approximation. For small basins with specific local conditions, this might not be the case especially for the quickly decaying part of the correlation functions connected to the parameter $k_{1}$. This is confirmed for two of the basins utilised here (a600101 and a605102 along branch 4). We interpret the large-scale component as a characteristic of the baseflow and seasonal climatic conditions for the region. The slowly decaying part of the correlation functions related to the parameter $k_{2}$ is rather stable. The weight coefficient $w$ balancing the local and regional influence seems also to be relatively constant for larger basins, while small ones may show deviations from this regional value possibly due to different baseflow contributions. The parameters $a, b, c$ and $d$ are all interpreted to be of a regional character for the time being.

\section{$7 \quad$ Discussion and conclusions}

The problem of mapping runoff characteristics has been divided into two steps in this paper. First, the first order moment (the long term mean value) is analysed and mapped applying an interpolation procedure for river runoff proposed by Gottschalk (1993b). In a second step a simple random model for the river instantaneous point runoff process normalised with respect to the long term mean is proposed, which allows the derivation of the time-space variancecovariance function of the inflow to the river network. This function is then used to predict how the temporal coefficient of variation develops from headwaters of the different river branches down to the river mouth. The runoff characteristics in a downstream point, here the first and second order moments, are thus derived by integration over the upstream contributing river network. The results are thus consistent in this respect and furthermore are able to reproduce the main features of the space-time covariance within a basin. The observation data are handled by a hydrological information system HydroDem (Leblois and Sauquet, 2000), which al- lows to display the results either in the form of area dependence of moments along the river branches to the basin outlet or as a map of the variation of the moments across the basin space.

The runoff variation across space is first of all explained by the variation in the average runoff formation. For the Moselle River basin (French part) studied here, the characteristic space scale of this process has been identified as $50 \mathrm{~km}$. The basic parameters in the stochastic model for the second order moment express the characteristic scales in time. Two characteristic temporal scales were identified - one related to the dynamics of the runoff formation process in the order of a day, $k_{1}$, and another related to the persistence in baseflow and climatic condition in the order of a month, $k_{2}$.

While the influence of the time scale on auto- and crosscorrelation functions is well in agreement between empirical and modelled data, the influence of the spatial scale, expressed as a "time of concentration" is more complex and not yet fully captured by the model. In this paper the "time of concentration" is a measure of the variance reduction in space, which corresponds to a similar reduction in time. A possible reason for the poorer fit might be the assumption of one global set of parameters for the whole Moselle basin. Further studies are needed to verify if allowing the parameters to vary between river branches (in particular the small time scale parameter $k_{1}$ and the weight coefficient $w$ balancing the influence of the two time scales) can improve the results. However, aggregation rules need to be elaborated for this purpose. Other critical points that need to be further elaborated are the present simplistic time-space correlation function Eq. (5) utilised here and the assumption about stationarity in time, neglecting seasonality.

Distributed modelling is an expanding activity in hydrology that lately also has been used for mapping purposes (e.g. Beldring et al., 2002). A fair comparison between the two approaches is possible, however, only if the diagnostics used for evaluating the performance of distributed models is extended to their ability of preserving time-space statistics and scale dependence. The preservation of time-space statistics and scale properties is of outmost importance for further developments for modelling and mapping of runoff for different durations as in this paper, and extremes (floods and drought) for different durations. 


\section{Appendix A}

Maps
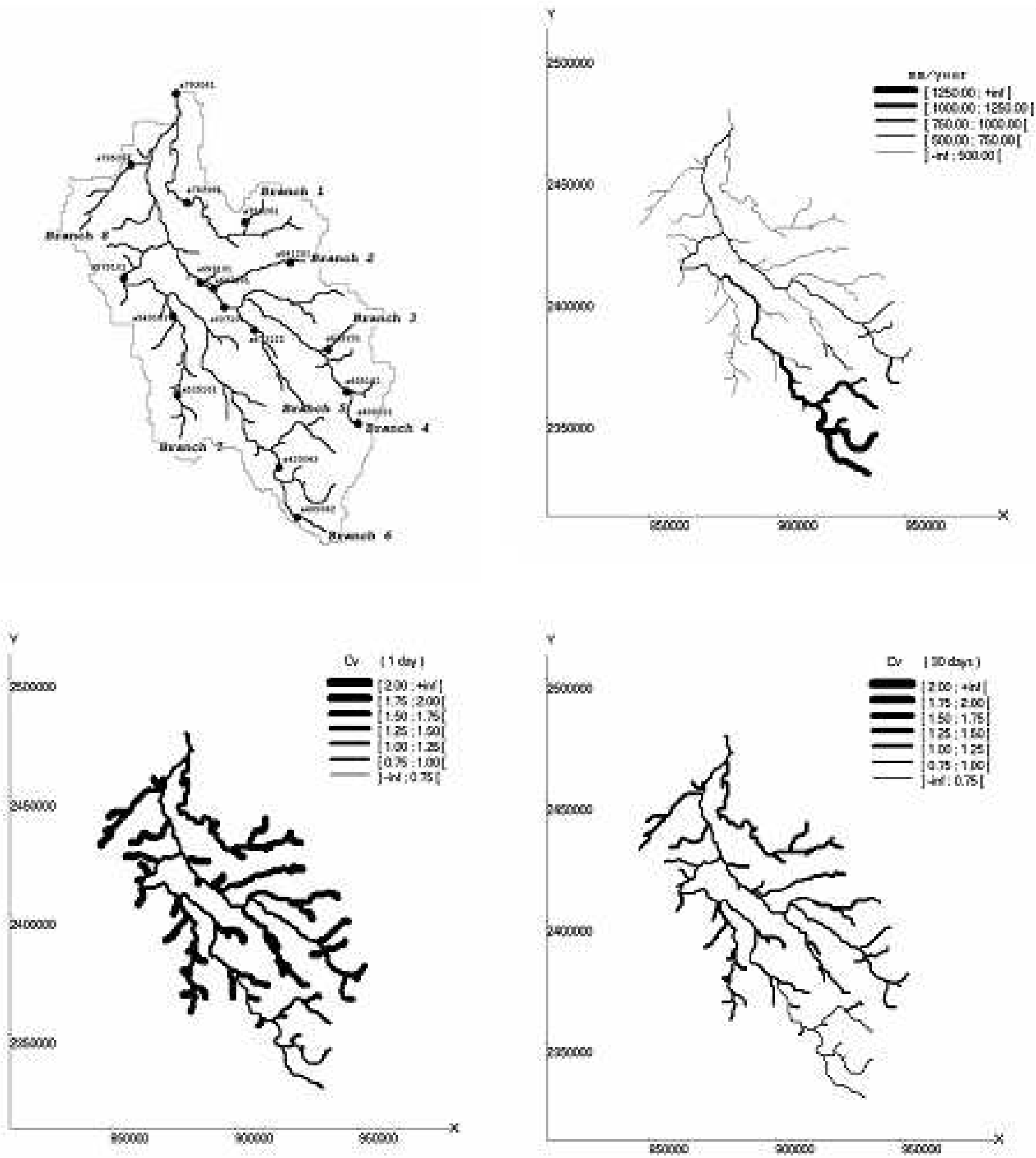

Fig. A1. 


\section{Appendix B}

B1 Variance function for a basin area $A$ and duration $D$ $\delta=D / k ; \eta=T_{c A} / k ; \tau^{\prime}=\tau / k ;$

$f_{1}(\lambda)=\frac{1}{\delta}\left(1+\frac{\lambda-\tau^{\prime}}{\delta}\right) ; \quad\left(\tau^{\prime}-\delta\right) \leq \lambda \leq \tau^{\prime}$

$f_{2}(\lambda)=\frac{1}{\delta}\left(1-\frac{\lambda-\tau^{\prime}}{\delta}\right) ; \quad \tau^{\prime}<\lambda \leq\left(\tau^{\prime}+\delta\right)$

i) $0<\delta \leq \eta$;

$$
\begin{aligned}
\gamma_{A}(\delta)= & 4 \sigma_{Z}^{2}(\delta \eta)^{-2}\left\{\frac{\delta^{2} \eta-\delta^{3} / 3}{2}+\frac{1}{2}\left(e^{(\delta-\eta)}\right.\right. \\
& \left.\left.+e^{-(\delta+\eta)}\right)+1-\delta-e^{-\eta}-e^{-\delta}\right\}
\end{aligned}
$$

ii) $\delta>\eta$;

$$
\begin{aligned}
\gamma_{A}(\delta)= & 4 \sigma_{Z}^{2}(\delta \eta)^{-2}\left\{\frac{\delta \eta^{2}-\eta^{3} / 3}{2}+\frac{1}{2}\left(e^{-(\delta-\eta)}\right.\right. \\
& \left.\left.+e^{-(\delta+\eta)}\right)+1-\eta-e^{-\eta}-e^{-\delta}\right\}
\end{aligned}
$$

B2 Auto-covariance function for a basin area $A$ and time lag $n D$

$\delta=D / k ; \eta=T_{c A} / k$

$f(\lambda)=\frac{1}{\delta}\left(1-n+\frac{\lambda}{\delta}\right) ;(n-1) \delta \leq \lambda \leq n \delta$

$f(\gamma)=\frac{1}{\delta}\left(1+n-\frac{\lambda}{\delta}\right) ; n \delta \leq \lambda \leq(n+1) \delta$

i) $(n+1) \delta \leq \eta$

$$
\begin{aligned}
\operatorname{Cov}_{A}(n \delta)= & 2 \sigma_{Z}^{2}(\delta \eta)^{-2}\left\{\delta^{2}(\eta-n \delta)+\left(e^{\delta / 2}-e^{-\delta / 2}\right)^{2}\right. \\
& \left.\left(\frac{1}{2} e^{-(n \delta+\eta)}+\frac{1}{2} e^{(n \delta-\eta)}-e^{n \delta}\right)\right\} \quad(\mathrm{B} 4 \mathrm{a})
\end{aligned}
$$

ii) $n \delta \leq \eta<(n+1) \delta$

$$
\begin{aligned}
\operatorname{Cov}_{A}(n \delta)= & 4 \sigma_{Z}^{2}(\delta \eta)^{-2}\left\{e^{-n \delta}\right. \\
& +\frac{1}{4}\left(e^{-((n-1) \delta+\eta)}+e^{((n-1) \delta-\eta)}\right. \\
& \left.+e^{(-(n+1) \delta+\eta)}+e^{(-(n+1) \delta+\eta)}\right) \\
& -\frac{1}{2}\left(e^{-(n-1) \delta}+e^{-(n+1) \delta}+e^{(n \delta-\eta)}\right. \\
& \left.+e^{-(n \delta+\eta)}\right)-\eta / 2+\eta^{3} / 12 \\
& +\delta(1+n) / 2+\left(\delta^{2} \eta\left(n^{2}+2 n-1\right)\right. \\
& -\delta \eta^{2}(n+1)-\delta^{3} \\
& \left.\left.\left(\frac{1}{3} n^{3}+n^{2}-n+\frac{1}{3}\right)\right) / 4\right\}
\end{aligned}
$$

iii) $(n-1) \delta \leq \eta<n \delta$

$$
\begin{aligned}
\operatorname{Cov}_{A}(n \delta)= & 4 \sigma_{Z}^{2}(\delta \eta)^{-2} \\
& \left\{e^{-n \delta}+\frac{1}{4}\left(e^{-((n-1) \delta+\eta)}+e^{((n-1) \delta-\eta)}\right.\right. \\
& \left.+e^{-((n-1) \delta-\eta)}+e^{(-(n+1) \delta+\eta)}\right) \\
& \frac{1}{2}\left(e^{-(n-1) \delta}+e^{-(n+1) \delta}\right. \\
& \left.+e^{(-n \delta+\eta)}+e^{-\left(n \delta_{\eta}\right)}\right)+\eta / 2-\eta^{3} / 12 \\
& +\delta(1-n) / 2-\left(\delta^{2} \eta\left(n^{2}-2 n+1\right)\right. \\
& -\delta \eta(n-1)-\delta^{3}\left(\frac{1}{3} n^{3}-n^{2}\right. \\
& \left.\left.\left.+n-\frac{1}{3}\right)\right) / 4\right\}
\end{aligned}
$$

iv) $(n-1) \delta>\eta$

$$
\begin{aligned}
\operatorname{Cov}_{A}(n \delta)= & \sigma_{Z}^{2}\left\{(\delta \eta)\left(1-e^{-\eta}\right)\left(e^{\delta / 2}-e^{-\delta / 2}\right)\right\}^{2} \\
& e^{-(n \delta-\eta)}
\end{aligned}
$$

B3 Covariance between two nested catchments $A_{1}$ and $A_{2}$ $\left(\mathrm{A}_{1}<\mathrm{A}_{2}\right)$

(Gottschalk, 1993a)

$\eta_{1}=T_{c A 1} / k ; \eta_{2}=T_{c A 2} / k$

$$
\begin{gathered}
f_{1}(\lambda)=\frac{1}{\eta_{2}}\left(1+\frac{\lambda}{\eta_{1}}\right) ; \quad-\eta_{1} \leq \lambda \leq 0 \\
f_{2}(\lambda)=\frac{1}{\eta_{2}} ; \quad 0 \leq \lambda \leq\left(\eta_{2}-\eta_{1}\right) \\
\begin{array}{c}
f_{3}(\lambda)=\frac{1}{\eta_{2}}\left(1-\frac{\lambda}{\eta_{1}}\right) ; \quad\left(\eta_{2}-\eta_{1}\right)<\lambda \leq \eta_{2} \\
\operatorname{Cov}\left(A_{1}, A_{2}, 0\right)=2 \sigma_{Z}^{2}\left(\eta_{1} \eta_{2}\right)^{-1}\left\{\eta_{1}+\frac{1}{2} e^{-\eta_{1}}\right. \\
+\frac{1}{2}\left(1-\eta_{1}+\eta_{2}\right)\left(e^{-\eta_{2}}\right. \\
\left.\left.-e^{-\left(\eta_{2} \eta_{1}\right)}\right)-\frac{1}{2}\right\}
\end{array}
\end{gathered}
$$


B4 Cross-covariance between two nested catchments $A_{1}$ and $\mathrm{A}_{2}\left(\mathrm{~A}_{1}<\mathrm{A}_{2}\right)$ for instantaneous runoff with lag $\tau^{\prime}$

$\eta_{1}=T_{c A 1} / k ; \eta_{2}=T_{c A 2} / k ;^{\prime}=\tau / k$

$$
\begin{aligned}
f_{1}(\lambda)= & \frac{1}{\eta_{2}}\left(1+\frac{\lambda-\tau^{\prime}}{\eta_{1}}\right) ; \quad\left(\tau^{\prime}-\eta_{1}\right) \leq \lambda \leq \tau^{\prime} \\
f_{2}(\lambda)= & \frac{1}{\eta_{2}} ; \quad \tau^{\prime} \leq \lambda \leq\left(\eta_{2}-\eta_{1}+\tau^{\prime}\right) \\
f_{3}(\lambda)= & \frac{1}{\eta_{2}}\left(1-\frac{\lambda-\tau^{\prime}}{\eta_{1}}\right) ; \\
& \left(\eta_{2}-\eta_{1}+\tau^{\prime}\right)<\lambda \leq\left(\eta_{2}+\tau^{\prime}\right)
\end{aligned}
$$

i) $\tau=0$;

$$
\begin{aligned}
\operatorname{Cov}\left(A_{1}, A_{2}, 0\right)= & 2 \sigma_{Z}^{2}\left(\eta_{1} \eta_{2}\right)^{-1} \\
& \left\{\eta_{1}+\frac{1}{2} e^{-\eta_{1}}+\frac{1}{2}\left(1-\eta_{1}+\eta_{2}\right)\right. \\
& \left.\left(e^{-\eta_{2}}-e^{-\left(\eta_{2}-\eta_{1}\right)}\right)-\frac{1}{2}\right\}
\end{aligned}
$$

ii) $0<\tau \leq \eta_{1}$

$$
\begin{aligned}
\operatorname{Cov}\left(A_{1}, A_{2}, \tau\right)= & 2 \sigma_{Z}^{2}\left(\eta_{1} \eta_{2}\right)^{-1} \\
& \left\{\left(\eta_{1}-\tau\right)+\frac{1}{2} e^{-\left(\eta_{1}-\tau\right)}\right. \\
& +\frac{1}{2}\left(1-\eta_{1}+\eta_{2}\right)\left(e^{-\left(\eta_{2}+\tau\right)}\right. \\
& \left.\left.-e^{-\left(\eta_{2}-\eta_{1}+\tau\right)}\right)-\frac{1}{2} e^{-\tau}\right\}
\end{aligned}
$$

iii) $\tau>\eta_{1}$;

$$
\begin{aligned}
\operatorname{Cov}\left(A_{1}, A_{2}, \tau\right)= & \sigma_{Z}^{2}\left(\eta_{1} \eta_{2}\right)\left\{e^{-\left(\eta_{2}-\eta_{1}\right)}-e^{-\eta_{2}}\right\} \\
& \left\{1-\left(1-\eta_{1}+\eta_{2}\right) e^{-\eta_{2}}\right\} e^{-\left(\tau-\eta_{2}\right)}
\end{aligned}
$$

B5 Cross-covariance between two nested catchments $A_{1}$ and $\mathrm{A}_{2}\left(\mathrm{~A}_{1}<\mathrm{A}_{2}\right)$ for runoff with time lag $\mathrm{nD}$

$\delta=D / k ; \eta_{1}=T_{c A 1} / k ; \eta_{2}=T_{c A 2} / k ;$

$f(\lambda)=\frac{1}{\delta}\left(1-n+\frac{\lambda}{\delta}\right) ;(n-1) \delta \leq \lambda \leq n \delta$
$f(\lambda)=\frac{1}{\delta}\left(1+n-\frac{\lambda}{\delta}\right) ; n \delta \leq \lambda \leq(n+1) \delta$ i) $\delta \leq \eta_{1}, n=0$

$$
\begin{aligned}
\operatorname{Cov}\left(A_{1}, A_{2}, 0\right)= & 2 \sigma_{Z}^{2}\left(\delta^{2} \eta_{1} \eta_{2}\right)^{-1} \\
& \left\{1+\delta^{2}\left(\eta_{1}-\delta / 3\right)+e^{-\left(\eta_{1}-\delta\right)}\right. \\
& -e^{-\delta}-(\delta+1) e^{-\eta_{1}} \\
& -\delta+\left(1-\eta_{1}+\eta_{2}\right)\left(e^{-\left(\delta+\eta_{2}\right)}\right. \\
& \left.-e^{-\left(\delta-\eta_{1}+\eta_{2}\right)}\right)-(1-\delta) \\
& \left(1-\eta_{1}+\eta_{2}\right) \\
& \left.\left(e^{-\eta_{2}}-e^{-\left(\eta_{2}-\eta_{1}\right)}\right)\right\}
\end{aligned}
$$

ii) $\delta>\eta_{1}, n=0$

$$
\begin{aligned}
\operatorname{Cov}\left(A_{1}, A_{2}, 0\right)= & 2 \sigma_{Z}^{2}\left(\delta^{2} \eta_{1} \eta_{2}\right)^{-1} \\
& \left\{1+\eta_{1}^{2}\left(\delta-\eta_{1} / 3\right)+e^{\left(\eta_{1}-\delta\right)}\right. \\
& -e^{-\delta}-(\delta+1) e^{-\eta_{1}}+\delta-2 \eta_{1} \\
& +\left(1-\eta_{1}+\eta_{2}\right)\left(e^{-\left(\delta+\eta_{2}\right)}\right. \\
& \left.-e^{-\left(\delta+\eta_{1}\right)}\right)-(1-\delta) \\
& \left(1-\eta_{1}+\eta_{2}\right)\left(e^{-\eta_{2}}\right. \\
& \left.\left.-e^{-\left(\eta_{2}-\eta_{1}\right)}\right)\right\}
\end{aligned}
$$

iii) $(n+1) \delta \leq \eta_{1}, n \geq 1$

$$
\begin{aligned}
\operatorname{Cov}\left(A_{1}, A_{2}, n D\right)= & 2 \sigma_{Z}^{2}\left(\delta^{2} \eta_{1} \eta_{2}\right)^{-1} \\
& \left\{\delta^{2}\left(\eta_{1}-n \delta\right)\right. \\
& +\frac{1}{2}\left(e^{\delta / 2}-e^{-\delta / 2}\right)^{2}\left[e^{-\left(n \delta-\eta_{2}\right)}\right. \\
& -e^{-n \delta}+\left(1-\eta_{1}+\eta_{2}\right)\left(e^{-\left(n \delta+\eta_{2}\right)}\right. \\
& \left.\left.\left.-e^{-\left(\eta_{2}-\eta_{1}+n \delta\right)}\right)\right]\right\} \quad(\mathrm{B} 10 \mathrm{c})
\end{aligned}
$$

iv) $(n-1) \delta>\eta_{1}, n>1$

$$
\begin{aligned}
\operatorname{Cov}\left(A_{1}, A_{2}, n D\right)= & \sigma_{Z}^{2}\left(\delta^{2} \eta_{1} \eta_{2}\right)^{-1}\left(e^{\delta / 2}-e^{-\delta / 2}\right)^{2} \\
& \left(e^{-\left(\eta_{2}-\eta_{1}\right)}-e^{\eta_{2}}\right)(1- \\
& \left.\left(1-\eta_{1}+\eta_{2}\right) e^{-\eta_{2}}\right) e^{-\left(n \delta-\eta_{2}\right)}(\mathrm{B} 10 \mathrm{~d})
\end{aligned}
$$

Acknowledgements. The authors thank G. Blöschl for his very constructive and valuable comments on the first version of the paper.

Edited by: A. Gelfan

\section{References}

Beldring, S., Roald, L. A. and Voks $\varnothing$, A.: Avrenningskart for Norge (Runoff map of Norway, in Norwegian), Norwegian Water and Energy Directorate Report, 2, 2002, Oslo, Norway, 2002. 
Gandin, L. S. and Kagan, P. L.: Statisticheskie metody dlya interpretatsii meteorologicheskykh dannykh. (Statistical methods for interpretation of meteorological data, in Russian), Gidrometeoizdat, Leningrad, 1976.

Gergov, G. I.: Zakonomernosti v izmenenii modulya stoka (Regularities in the variation of specific runoff, in Russian ), Meteorologiya i Gidrologiya 8, 75-81, 1972.

Ghosh, B.: Random distances within a rectangle and between two rectangles, Bull. Calcutta Math. Soc., 43, 1951.

Gottschalk, L.: Correlation and covariance of runoff, Stochastic Hydrology and Hydraulics, 7, 85-101, 1993a.

Gottschalk, L.: Interpolation of runoff applying objective methods, Stochastic Hydrology and Hydraulics, 7, 269-281, 1993b.

Gottschalk, L.: Methods for analysing variability, in: Encyclopaedia of Hydrological Sciences, edited by: Anderson, M. G. and McDonnell, J. J., John Wiley \& Sons, 1, 6, 95-122, 2005.

Gupta, V. K. and Waymire, E.: Multiscaling properties of spatial rainfall and river flow distribution, J. Geophys. Res., 95(D3), 1999-2009, 1990.

Krasovskaia, I. and Gottschalk, L.: Stability of river flow regimes, Nordic Hydrology, 23, 137-154, 1992.
Leblois, E. and Sauquet, E.: Grid elevation models in hydrology - Part 1: Principles and a literature review; Part 2: HydroDem, User's manual, Cemagref, Technical Notes, Lyon, 80 pp., 2000.

Matérn, B.: Spatial Variation, Meddelanden från Statens Skogsforskiningsinstitut, 49(5), 1960.

Press, W. H., Flannery, B. P., Teukolsky, S. A., and Vetterling, W. T.: Numerical recipes in Pascal, Cambridge University Press, N.Y., 1992.

Sauquet, E.: Une cartographie des écoulements annuels et mensuels d'un grand basin versant structurée par la topologie du réseau hydrographique, Thèse - Institut National Polytechnique de Grenoble 1, 356 pp., 2000.

Sauquet, E., Gottschalk, L., and Leblois, E.: Mapping average annual runoff: A hierarchical approach applying a stochastic interpolation scheme, Hydrol. Sci. J., 45(6), 799-815, 2000.

Singh, V. P.: Hydrological systems: Rainfall-Runoff Modeling, Prentice Hall, Englewood Cliffs, New Jersey, 480 pp., 1988.

Tukey, J. W.: Bias and confidence in not-quite large samples, (Abstract), Ann. Math. Stat., 29, 614, 1961.

Vanmarcke, E.: Random Fields. Third printing, MIT press, Cambridge, Mass., 382 pp., 1988. 